\title{
Publisher Correction: m6A modification controls the innate immune response to infection by targeting type I interferons
}

Roni Winkler, Ella Gillis, Lior Lasman, Modi Safra, Shay Geula, Clara Soyris, Aharon Nachshon ID, Julie Tai-Schmiedel, Nehemya Friedman, Vu Thuy Khanh Le-Trilling, Mirko Trilling, Michal Mandelboim, Jacob H. Hanna (D), Schraga Schwartz and Noam Stern-Ginossar (D)

Correction to: Nature Immunology https://doi.org/10.1038/s41590-018-0275-z (2018), published online 17 December 2018.

In the version of this article initially published, the penultimate sentence of the abstract included a typographical error ('cxgenes'). The correct word is 'genes'. The error has been corrected in the HTML and PDF version of the article.

Published online: 11 January 2019

https://doi.org/10.1038/s41590-019-0314-4 